\title{
A large family with hereditary spastic paraparesis due to a frame shift mutation of the spastin (SPG4) gene: association with multiple sclerosis in two affected siblings and epilepsy in other affected family members
}

\author{
S H Mead, C Proukakis, N Wood, A H Crosby, G T Plant, T T Warner
}

\begin{abstract}
Hereditary spastic paraparesis (HSP) is a clinically and genetically heterogeneous neurodegenerative disorder characterised by progressive lower limb spasticity and weakness. Some forms have been associated with white matter lesions and complex phenotypes. This study was prompted by the diagnosis of multiple sclerosis (MS) in two sisters from a large pedigree with hereditary spastic paraparesis. Twelve affected members of the extended family were examined (MRI and EEG were carried out and evoked potentials measured in five), and historical information was obtained from six affected deceased persons. The inherited disease phenotype was confirmed as autosomal dominant hereditary spastic paraparesis associated with epilepsy in four affected persons. None of the extended family had evidence of MS. Genetic analysis of the family has shown linkage to chromosome $2 p$ and sequencing of the spastin gene has identified a 1406delT frameshift mutation in exon 10. This kindred demonstrates the clinical heterogeneity of HSP associated with spastin mutations. The possible relevance of the concurrence of HSP and MS in the sib pair is discussed.

(F Neurol Neurosurg Psychiatry 2001;71:788-791)
\end{abstract}

Keywords: hereditary spastic paraparesis; multiple sclerosis; spastin

Hereditary spastic paraparesis (HSP) is a disease characterised by progressive lower limb spasticity and weakness. Autosomal dominant HSP is the most common form and eight genetic loci have been identified. It is estimated that around $40 \%$ of autosomal dominant HSP kindreds are linked to a locus on chromosome $2 \mathrm{p}$ (SPG4). ${ }^{12}$ There is considerable phenotypic heterogeneity within pedigrees linked to the SPG4 locus, with evidence of epilepsy, cognitive impairment, and neurological abnormalities from birth in some pedigrees. ${ }^{34}$ Recently, mutations have been identified in the spastin gene in families with SPG4 linked HSP. $^{5-8}$

There is evidence of considerable genetic susceptibility in multiple sclerosis (MS), with involvement of HLA haplotypes and some uncharacterised genetic loci. ${ }^{9}$ Our neurogenetic report was prompted by the identification of two siblings with clinical history and investigation findings consistent with the concurrence of both MS and HSP. Our aims were to characterise the clinical phenotype in the extended family and determine the genetic abnormality to clarify any susceptibility relation between HSP and MS.

\section{Methods}

The family was approached through the proband (fig 1). Informed written consent was obtained from all participants. Twenty six members of the extended family were examined by one of us (SHM). Diagnosis of HSP was based on established criteria of lower limb hyperreflexia plus either progressive spastic gait abnormality, bilateral extensor plantars, or bilateral ankle clonus. ${ }^{10}$ Diagnosis of MS was based on established criteria (table 1). ${ }^{11}$ Five members of the family were admitted to the National Hospital for Neurology for detailed investigation and a further two members had brain MRI as outpatients.

Blood was obtained from family members after informed consent and DNA was extracted using standard procedures. Linkage analysis using microsatellite markers around the SPG4 locus was performed. For one affected member with affected offspring (IV-10), the 17 exons of the SPG4 gene were amplified together with the flanking intronic regions using novel primers (Proukakis et al, unpublished data). Polymerase chain reaction (PCR) products were sequenced directly on an ABI377 automated DNA sequencer. To confirm the mutation, PCR products obtained from genomic DNA were subcloned (TOPO-TA cloning kit, Invitrogen) and products derived from both parental chromosomes sequenced. Mutation 


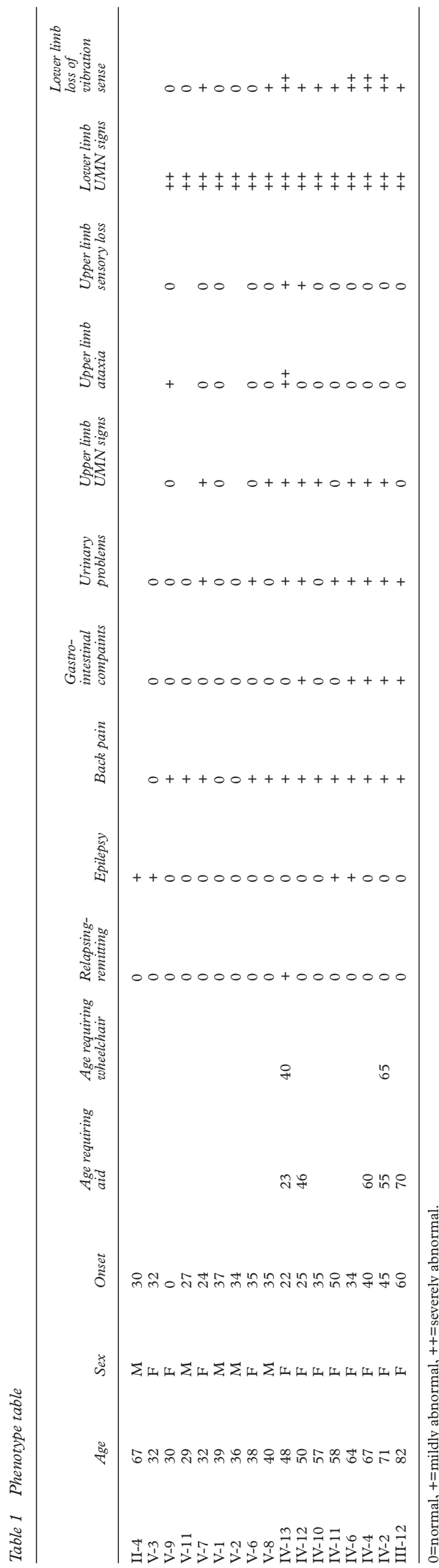

detection in other family members was performed by PCR amplification and sequencing of exon 10 .

\section{Results}

PROBAND CASE HISTORY (IV-13)

The proband presented at the age of 22 with rapid onset of visual disturbance. On examination she had impaired visual acuity, an incongruous left sided hemianopia, mild dysarthria, and impaired joint position sense in the left hand. These signs improved slowly over 6 weeks. At 3 months she had a residual left hemianopia, impaired colour vision, and a mild left hemiparesis. Fifteen years later she developed over 2 weeks a loss of balance, slurring dysarthria, and incoordination of her right hand. Examination in hospital recorded additional signs of a spastic paraparesis, with brisk lower limb reflexes and extensor plantars. A diagnosis of multiple sclerosis was made and intravenous methylprednisolone was given with some response.

Over the subsequent years her spastic gait deteriorated with increasing spasticity, eventually limiting her to a wheelchair. She developed urge incontinence, nocturia, and severe pains related to leg muscle spasms. On examination, aged 47, she had a left relative afferent pupillary defect with an acuity of $3 / 24,3 / 24$, and severely impaired colour vision. Pursuit eye movements were broken and jaw jerk was brisk. Tone was slightly increased in the arms but power was full with only a mild cerebellar ataxia. Tendon reflexes in the upper limb were brisk. In the lower limb there was markedly increased tone with sustained ankle clonus and hip flexion weakness of $4 / 5$. Deep tendon reflexes were brisk with extensor plantars responses. Brain MRI was markedly abnormal (fig 2). Analysis of CSF demonstrated oligoclonal immunoglobulin bands that were absent in serum.

PROBAND'S SISTER'S CASE HISTORY (IV-12)

The proband's sister presented aged 25 when she began to drag her left foot on the ground and she complained of a tight, heavy sensation affecting the upper thighs, back, and shoulders. The symptoms gradually progressed involving both lower limbs, although the left leg remained worse than the right. Her balance

became impaired, and she was unable to stand on one leg. She also had been diagnosed with anxiety depression. Aged 50, she was unable to walk outside more than $100 \mathrm{~m}$ with a walking stick and climbing stairs was difficult. Examination at age 50 showed brisk upper limb reflexes with bilateral Hoffman's sign. She had a spastic paraparesis with hypertonicity of both legs, ankle clonus, and a mild pyramidal type weakness. There were distal sensory abnormalities affecting all modalities in all four limbs. A brain MRI showed white matter lesions (fig 2).

Analysis of CSF showed the presence of oligoclonal immunoglobulin bands, which were absent in serum. 


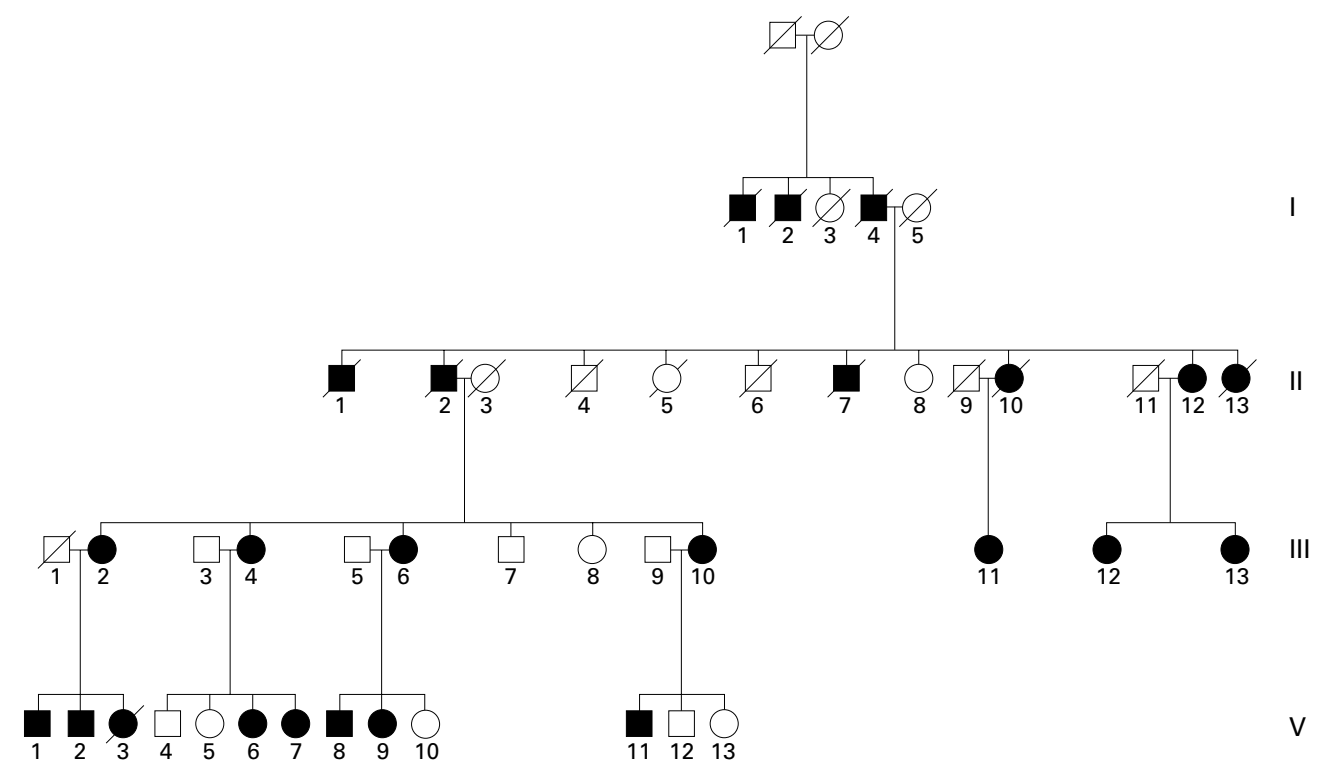

Figure 1 Pedigree. Filled circles represent affected people. Examined people are detailed in table 1. The details have been modified to preserve anonymity.

THE EXTENDED FAMILY

Inheritance of HSP was compatible with an autosomal dominant trait with no evidence of incomplete penetrance. The mean age of symptom onset was 36 (range 22 to 60) excluding one patient with abnormalities from birth. None of the extended family had a clinical history or examination findings suggestive of additional multiple sclerosis. There were considerable variations in the age of onset, nature, and severity of the disease within the family. Gastrointestinal complaints consisted of abdominal pain and constipation. Urinary complaints were of urge incontinence. There was no evidence of any presenile cognitive impairment (three had a detailed neuropsychological assessment).

Four of the family had a history of seizures that varied in type and severity. Descendants of
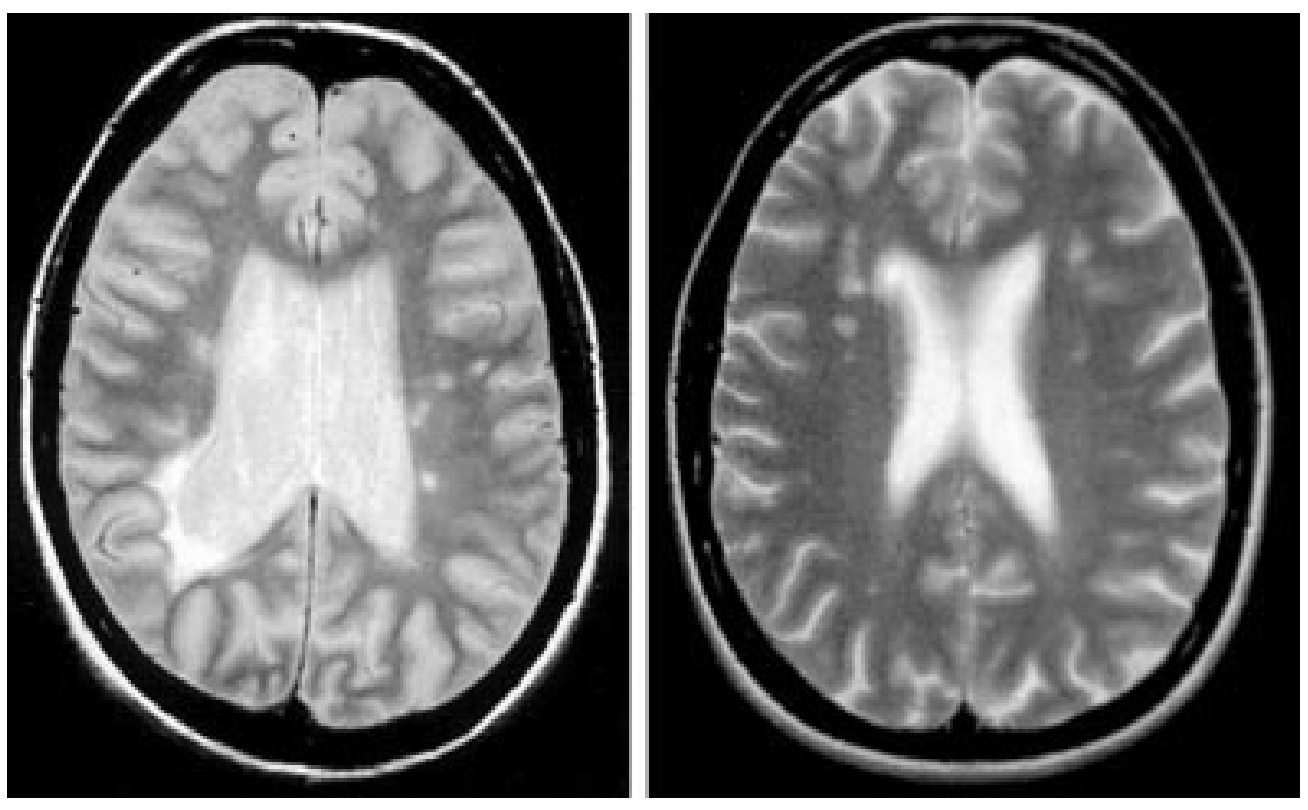

Figure 2 (Left) T2 weighted axial brain MRI showing large right periventricular focal white matter high signal in patient IV-13. (Right) T1 weighted axial brain MRI showing frontal focal white matter lesions in patient IV-12.

II-4 described this member's lifelong generalised tonic-clonic seizures with onset at age 12 . Patient IV-6 had generalised tonic-clonic seizures between the ages of 14 and 24. Patient IV-11 had a high frequency of seizures between the ages of 8 months and 5 years. Occasionally these consisted of tonic spasms and cyanosis, at other times there were jerking movements of the limbs. The mother of patient $\mathrm{V}-3$ gave a history likely to represent HSP in her daughter, by describing an abnormal gait similar to the rest of the family with onset at the age of 30 . She had episodic attacks that began in her early teens and continued at a frequency of 3 to 4 attacks a year. At the age of 32 she suddenly began to behave strangely, complaining of "filthy language" and being paralysed. A diagnosis of convulsive status epilepticus was made in hospital. She died after asystolic arrest. . 
GENETIC ANALYSIS

Linkage to the SPG4 locus was detected and sequencing disclosed a heterozygous one base pair deletion in exon 10 (1406delT) leading to frame shift (aa427-436) and premature termination (437stopcodon). The sequence was confirmed after subcloning. The presence of this mutation was confirmed in the two sisters with MS (IV-12 and IV-13).

\section{Discussion}

This report describes a large family with autosomal dominant HSP caused by a deletion in exon 10 of the spastin gene leading to a premature stop codon. The resulting transcript is predicted to produce a truncated spastin protein lacking the AAA cassette. This mutation has been reported in one other family ${ }^{7}$ with a clinical phenotype of pure HSP with no epilepsy. Four of our affected family members have a clinical history consistent with epilepsy not found in any unaffected family member. There was no consistency in the phenomenology of these seizures and there is no EEG confirmation of this diagnosis. Epilepsy has previously been reported in otherwise pure autosomal dominant $\mathrm{HSP}^{3}$ More recently a nonsense mutation in exon 3 of SPG4 $(702 \mathrm{C}>\mathrm{T})$ has been found in a previously described kindred with autosomal dominant HSP complicated by epilepsy and cognitive impairment and dementia. ${ }^{12}$ Our report is thus the second of a spastin mutation associated with epilepsy.

The proband (IV-13) had clinical findings consistent with the concurrence of relapsingremitting MS (fitting established criteria for clinically definite $M^{11}$ ) and HSP. Her sister, however, (IV-12) has not had clear cut relapses and remissions of MS but had clinical and investigation findings inconsistent with a diagnosis of HSP alone-namely, asymmetry of spasticity in the lower limb, distal sensory abnormalities in all four limbs, psychiatric morbidity, white matter lesions on brain MRI, and CSF oligoclonal bands. These findings are best explained by a combination of HSP and MS (fitting criteria for laboratory supported definite MS).

The prevalence of HSP has been estimated at 10:100 000 in the United Kingdom. ${ }^{10}$ The prevalence of MS in south east England (where the two sisters have spent their adult life) is around 1:1000. ${ }^{13} \mathrm{~A}$ chance association in an affected sib pair is therefore unlikely. Futhermore, in a large European case series of chromosome 2 linked HSP there is reference to a 42 year old woman with the HSP disease haplotype and a history of relapsing motor weakness, together with MRI and CSF findings consistent with MS. ${ }^{14}$ If the concurrence of MS and HSP is not coincidence, the spastin mutation may be a predisposing factor for MS. Possible associations between Leber's hereditary optic neuropathy (LHON) and $\mathrm{MS},{ }^{6}$ and also neurofibromatosis and MS have been reported. ${ }^{5}$ Hereditary spastic paraparesis with mutation in the spastin gene may be tentatively added to this group, subject to confirmation by finding additional patients with both conditions.

This family demonstrates the significant clinical heterogeneity that exists for spastin associated HSP, even in families with the same mutation. The association of HSP and MS is tantalising and should prompt others to seek further examples of such a link.

1 Reid E, Grayson C, Rubinsztein DC, et al. Subclinical cognitive impairment in autosomal dominant "pure" hereditary spastic paraplegia [letter]. F Med Genet 1999;36:797-8.

2 McDermott C, White K, Bushby K, et al. Hereditary spastic paraparesis: a review of new developments. $\mathcal{F}$ Neurol Neurosurg Psychiatry 2000;69:150-60.

3 Webb S, Flanagan N, Callaghan N, et al. A family with hereditary spastic paraparesis and epilepsy. Epilepsia 1997; 38:495-9.

4 White $\mathrm{KD}$, Ince $\mathrm{PG}$, Lusher $\mathrm{M}$, et al. Clinical and pathologic findings in hereditary spastic paraparesis with spastin mutation. Neurology 2000;55:89-94.

5 Ferner RE, Hughes RA, Johnson MR. Neurofibromatosis 1 and multiple sclerosis. F Neurol Neurosurg Psychiatry 1995; 58:582-5.

6 Harding AE, Sweeney MG, Miller DH, et al. Occurrence of a multiple sclerosis-like illness in women who have a Leber's hereditary optic neuropathy mitochondrial DNA er's hereditary optic neuropathy

7 Lindsey JC, Lusher ME, McDermott CJ, et al. Mutation analysis of the spastin gene (SPG4) in patients with hereditary spastic paraparesis. 7 Med Genet 2000;37:759-65.

8 Fonknechten N, Mavel D, Byrne P, et al. Spectrum of SPG4 mutations in autosomal dominant spastic paraplegia. Hum Mol Genet 2000;9:637-44.

9 Ebers GC, Kukay K, Bulman DE, et al. A full genome search in multiple sclerosis. Nat Genet 1996;13:472-6.

10 Harding AE. Hereditary spastic paraplegias. Semin Neurol 1993;13:333-6.

11 Poser CM, Paty DW, Scheinberg L, et al. New diagnostic criteria for multiple sclerosis: guidelines for research protocols. Ann Neurol 1983;13:227-31.

12 Heinzlef O, Paternotte C, Mahieux F, et al. Mapping of a complicated familial spastic paraplegia to locus SPG4 on chromosome 2p. F Med Genet 1998;35:89-93.

13 Mumford CJ, Fraser MB, Wood NW, et al. Multiple sclerosis in the Cambridge health district of East Anglia. $\mathcal{F}$ Neurol sis in the Cambridge health district of
Neurosurg Psychiatry 1992;55:877-82.

14 Durr A, Davoine CS, Paternotte C, et al. Phenotype of autosomal dominant spastic paraplegia linked to chromosome 2. Brain 1996;119:1487-96. 